\title{
Systematic review and meta-analysis of uniportal versus multiportal video-assisted thoracoscopic lobectomy for lung cancer
}

\author{
Christopher G. Harris ${ }^{1}$, Rebecca S. James ${ }^{1,2}$, David H. Tian ${ }^{1,3}$, Tristan D. Yan ${ }^{1,4,5}$, Mathew P. Doyle ${ }^{6}$, \\ Diego Gonzalez-Rivas ${ }^{7}$, Christopher $\mathrm{Cao}^{1,6}$ \\ ${ }^{1}$ The Collaborative Research (CORE) Group, Macquarie University, Sydney, Australia; ${ }^{2}$ The Sydney Children's Hospital, Sydney, Australia; ${ }^{3}$ Royal \\ North Shore Hospital, Sydney, Australia; ${ }^{4}$ Department of Cardiothoracic Surgery, Royal Prince Alfred Hospital, Sydney, Australia; ${ }^{5}$ University of \\ Sydney, Sydney, Australia; ${ }^{6}$ Department of Cardiothoracic Surgery, St George Hospital, Sydney, Australia; ${ }^{7}$ Department of Thoracic Surgery (Coruña \\ University Hospital) and Minimally Invasive Thoracic Surgery Unit (UCTMI), Coruña, Spain \\ Correspondence to: Christopher Cao. The Collaborative Research (CORE) Group, Macquarie University, Sydney, Australia. Email: chriscao@annalscts.com.
}

\begin{abstract}
Background: Uniportal video-assisted thoracoscopic surgery (VATS) has emerged as a less invasive alternative to the conventional multiportal approach in the treatment of lung cancer. The benefits of this uniportal technique have not yet been characterized in patients undergoing VATS lobectomy. This metaanalysis aimed to compare the clinical outcomes of uniportal and multiportal VATS lobectomy for patients with lung cancer.

Methods: A systematic review was conducted using seven electronic databases. Endpoints for analysis included perioperative mortality and morbidity, operative time, length of hospital stay, perioperative blood loss, duration of postoperative drainage and rates of conversion to open thoracotomy.

Results: Eight relevant observational studies were identified and included for meta-analysis. Results demonstrated a statistically significant reduction in the overall rate of complications, length of hospital stay and duration of postoperative drainage for patients who underwent uniportal VATS lobectomy. There were no significant differences between the two treatment groups in regard to mortality, operative time, perioperative blood loss and rate of conversion to open thoracotomy.

Conclusions: The present meta-analysis demonstrated favourable outcomes for uniportal VATS lobectomy in the treatment of lung cancer compared to the conventional multiportal approach. However, long-term follow-up data is still needed to further characterize the benefits of the uniportal approach.
\end{abstract}

Keywords: Uniportal; video-assisted thoracoscopic surgery (VATS); lobectomy; lung cancer; meta-analysis

Submitted Mar 15, 2016. Accepted for publication Mar 24, 2016.

doi: $10.21037 /$ acs.2016.03.17

View this article at: http://dx.doi.org/10.21037/acs.2016.03.17

\section{Introduction}

Over the past two decades, surgical management of lung cancer has been characterized by the emergence of novel minimally invasive surgical techniques. Video-assisted thoracoscopic surgery (VATS) has been shown to be associated with superior perioperative outcomes when compared to open thoracotomy, with numerous metaanalyses demonstrating reduced complication rates, shorter hospital stay and improved long-term survival (1-3). In recent years, a uniportal VATS technique has emerged as an even less invasive alternative to the conventional multiportal approach $(4,5)$. Since its adoption by thoracic surgeons, there have been numerous reports on the feasibility of this approach in the surgical management of lung and mediastinal tumors (5-7).

In addition to the reduced number of surgical incisions, institutional reports have demonstrated a number of potential advantages of the uniportal VATS technique. These included a significant reduction in postoperative pain $(8,9)$, paresthesia (10-12) and improved patient satisfaction $(11,13,14)$. Despite these encouraging results, comparative 
clinical outcomes of uniportal versus multiportal VATS remain uncertain. The present systematic review and metaanalysis aimed to compare uniportal VATS to conventional multiportal VATS in the context of lobectomy for lung cancer. Endpoints included mortality, operative time, length of hospital stay, perioperative blood loss and duration of postoperative drainage, as well as complication rates and rates of conversion to open thoracotomy.

\section{Methods}

\section{Search strategy and selection criteria}

Electronic searches were performed using Ovid Medline, Embase, PubMed, the Cochrane Central Register of Controlled Trials (CCTR), Cochrane Database of Systematic Reviews (CDSR), ACP Journal Club and Database of Abstracts of Reviews of Effects (DARE) from their inception date to January 2016. In order to maximize the sensitivity of the search and identify all relevant studies, we used the terms 'uniport ${ }^{*}$ ' or 'single-port' or 'single port' or 'single-incision' or 'single incision' combined with 'VATS' or 'thoracosp"' or 'video-assisted' or 'video assisted', either as key words or MeSH terms. Following initial screening based on abstracts, the full texts of potentially relevant articles were obtained. The reference lists of all retrieved articles were examined in order to identify additional potentially relevant studies.

Eligible comparative studies for the present systematic review and meta-analysis included those in which survival data was available for patients with a diagnosis of lung cancer treated by lobectomy in uniportal and multiportal VATS cohorts. Indications for surgery other than for lung malignancy, such as mediastinal tumors, primary spontaneous pneumothorax and hyperhidrosis, were excluded. In cases where institutions have published duplicated trials with accumulating patient cohorts, only the most recent and complete study was included for appraisal. All studies were limited to human subjects. Case reports, conference abstracts, editorials, expert opinions and commentaries were excluded. Review articles were also excluded due to possible duplication of results and publication bias.

\section{Data extraction and critical appraisal}

All data were extracted from article texts, tables and figures. Two investigators (C.G.H. and R.S.J.) independently reviewed each retrieved article. Any inconsistencies between the two reviewers were resolved by discussion and consensus. The final results were reviewed by the senior investigators (T.D.Y. and C.C.).

\section{Statistical analysis}

Meta-analysis was performed by combining the results of outcome variables. Data were summarized as standard mean difference, with overall weighted mean presented where appropriate. $\mathrm{I}^{2}$ statistic was used to estimate the percentage of total variation across studies, due to heterogeneity rather than chance. An $\mathrm{I}^{2}$ value of greater than $50 \%$ was considered substantial heterogeneity. If there was substantial heterogeneity, the possible clinical and methodological reasons for this were explored qualitatively. In the present meta-analysis, the results using the random-effects model were presented to take into account the possible clinical diversity and methodological variation amongst studies. Specific analyses considering confounding factors were not possible because raw data were not available. All $\mathrm{P}$ values were 2 -sided. A significant difference was defined as $\mathrm{P}<0.05$. Statistical analysis was conducted with Review Manager Version 5.3 (Cochrane Collaboration, Software Update, Oxford, UK).

\section{Results}

\section{Quantity and quality of trials}

A total of 1,051 references were identified by the electronic search strategy. After excluding duplicate or irrelevant articles, 38 references were retrieved for further evaluation. Manual searching of the reference lists of these retrieved articles did not identify any additional relevant studies. After applying the selection criteria and excluding studies that compared uniportal and multiportal VATS for indications other than lung cancer, eight studies remained for assessment. All of these studies were observational studies and deemed suitable for quantitative meta-analysis. The study selection process is summarized in Figure 1 according to the PRISMA statement (15). A summary of the study characteristics is presented in Table 1. Overall, a total of 1,850 patients were compared, including 627 patients who underwent uniportal VATS and 1,223 patients who underwent multiportal VATS. Three studies reported propensity-matched data (20-22). Two of these studies presented data from both unmatched and propensity- 


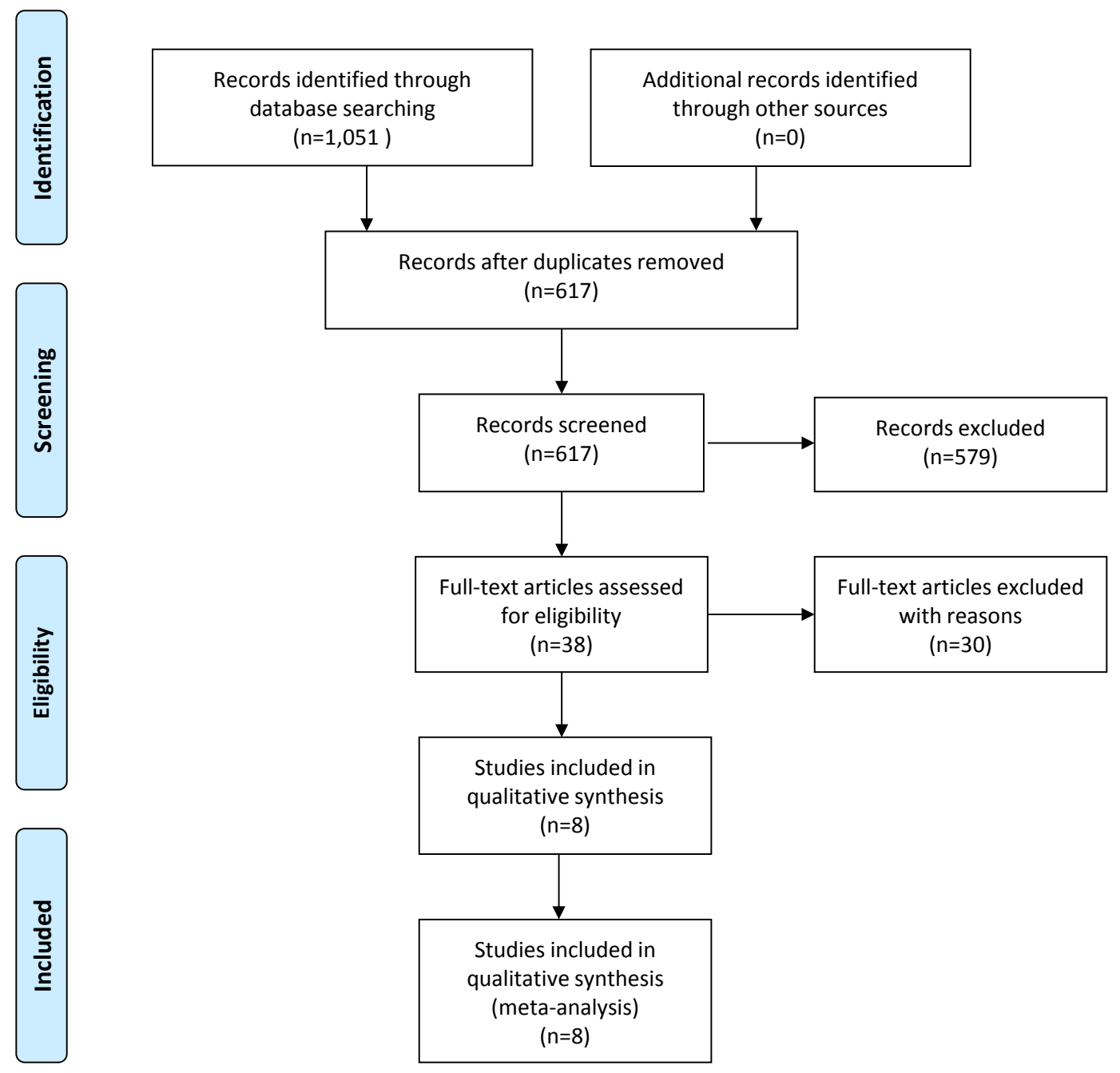

Figure 1 PRISMA flow chart for literature search.

\begin{tabular}{|c|c|c|c|c|c|}
\hline Authors & Publication & Study period & Indication & Uniportal (n) & Multiportal [n] \\
\hline Chung (16) & 2015 & 2013-2014 & Lung cancer & 90 & 60 [2-3] \\
\hline Li (18) & 2013 & 2011-2013 & Lung cancer & 87 & 75 [3] \\
\hline Liu (19) & 2016 & 2005-2014 & NSCLC & 149 & 389 [NR] \\
\hline Shen (21) & 2016 & 2013-2014 & Lung cancer & 100 & 100 [3] \\
\hline Wang (22) & 2015 & 2005-2013 & Lung cancer & 50 & 183 [2-3] \\
\hline Zhu (23) & 2015 & 2014-2014 & NSCLC & 33 & 49 [3] \\
\hline
\end{tabular}




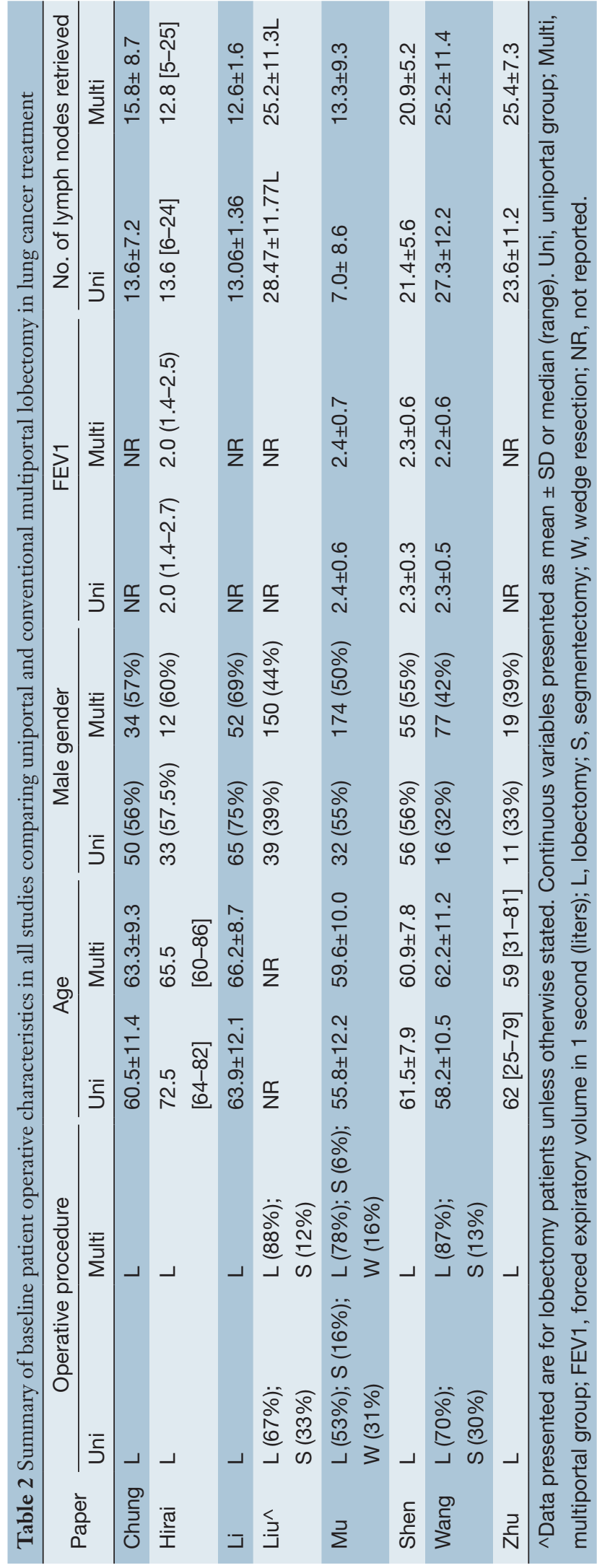

matched cohorts $(20,22)$ and unmatched data from these studies is presented in Tables 1-3.

\section{Patient operative and histopathological details}

A summary of the patient operative details is presented in Table 2 and a summary of patient histopathological details is summarized in Table 3. See Table S1 for a summary of patient comorbidities in all included studies.

\section{Assessment of operative outcomes}

There was a statistically significant reduction in the length of hospital stay for patients who underwent uniportal VATS lobectomy compared to the multiportal approach (6.2 \pm 2.6 vs. $6.7 \pm 3.4$ days, $\mathrm{P}<0.0001$, Figure 2). There was also a statistically significant reduction in the duration of postoperative drainage for the uniportal group $(4.5 \pm 2.2$ vs. 5.4 \pm 2.9 days, $\mathrm{P}=0.0006$, Figure 3). In regard to overall morbidity as reported by the included studies, there was a statistically significant reduction in the incidence of overall morbidities for patients undergoing uniportal VATS lobectomy compared to the multiportal approach $(12.0 \%$ vs. $13.7 \%, \mathrm{P}=0.009$, Figure 4).

There were no significant differences between uniportal versus multiportal VATS in regard to operative time (155.8 \pm 53.8 vs. $167 \pm 64.6$ minutes, $\mathrm{P}=0.69)$, perioperative blood loss $(86.3 \pm 76.2$ vs. $82.4 \pm 74 \mathrm{~mL}, \mathrm{P}=0.63)$ or rate of conversion to open thoracotomy ( $3.6 \%$ vs. $2.6 \%, \mathrm{P}=0.83)$. It should be noted that there were no perioperative mortalities in any patients who underwent uniportal VATS and only one patient who underwent the multiportal approach (16). Long-term clinical outcomes were not reported in any of the studies.

When propensity-matched data were analyzed, there were no statistically significant differences in operation time, length of hospital stay, perioperative blood loss, duration of postoperative drainage, rate of conversion to thoracotomy or overall morbidity. A summary of these findings is presented in Table 4 for all included studies and Table 5 for the studies with propensity-matched data.

\section{Discussion}

The present systematic review demonstrated that uniportal VATS was associated with a statistically significant shorter duration of chest tube drainage ( $4.5 \pm 2.2 v s .5 .3 \pm 2.9$ days), shorter hospital stay $(6.2 \pm 2.6$ vs. $6.7 \pm 3.4$ days $)$ and lower 


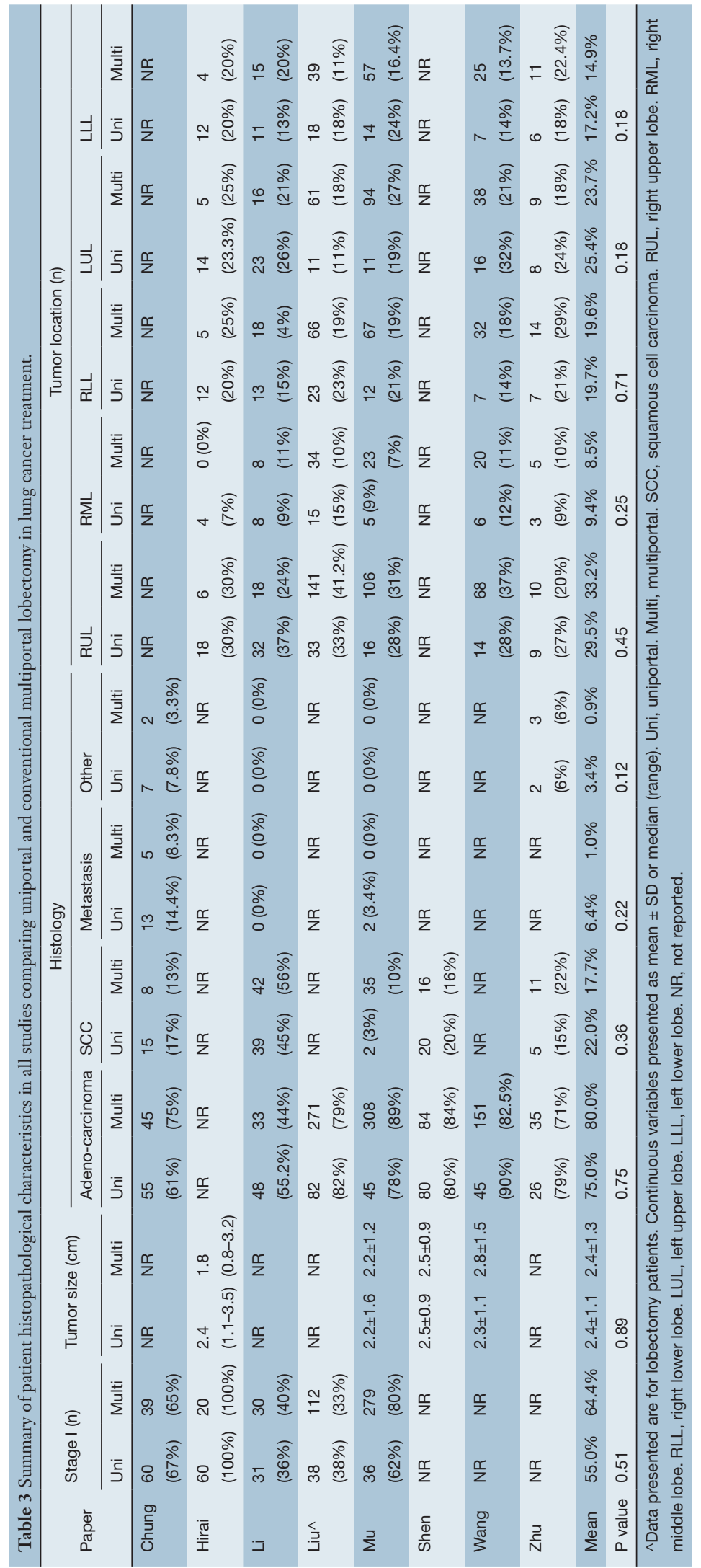




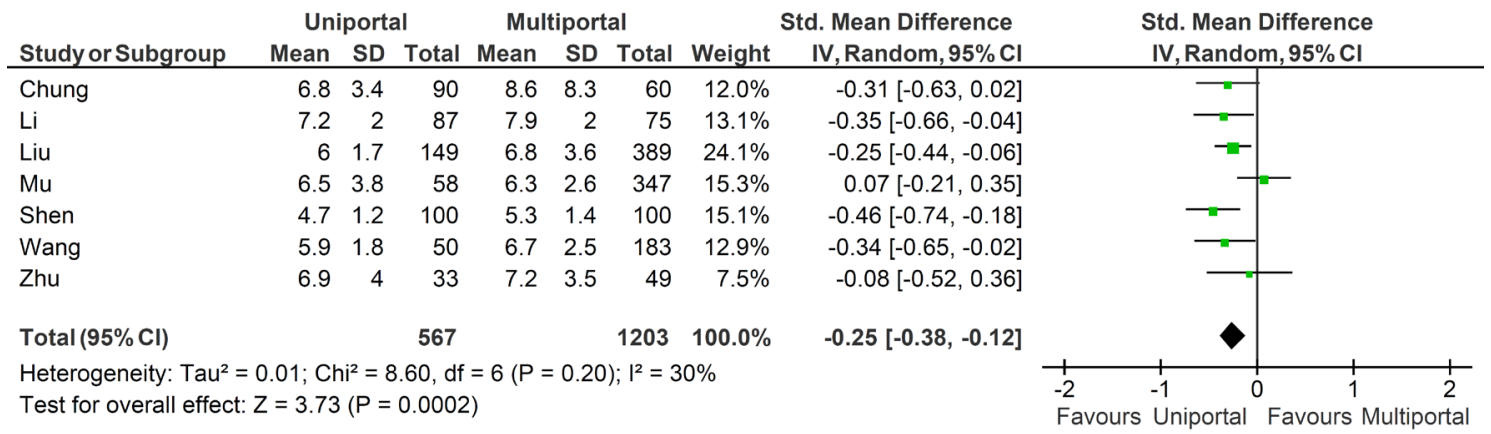

Figure 2 Forest plot of length of stay for uniportal and multiportal groups. The estimate of the mean difference of each study corresponds to the middle of the squares and the horizontal line shows the $95 \%$ confidence interval (CI). On each line, the mean and standard deviations are shown for both treatment groups. The sum of the statistics, along with the summary standardized mean difference, is represented by the middle of the solid diamonds. A test of heterogeneity between the trials within a subgroup is given below the summary statistics.

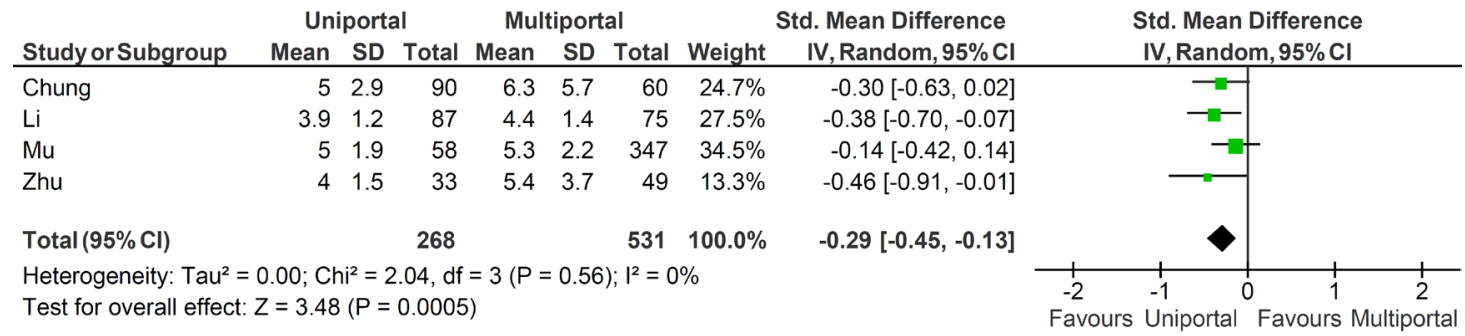

Figure 3 Forest plot of duration of postoperative drainage for uniportal and multiportal groups. The estimate of the mean difference of each study corresponds to the middle of the squares and the horizontal line shows the $95 \%$ confidence interval (CI). On each line, the mean and standard deviations are shown for both treatment groups. The sum of the statistics, along with the summary standardized mean difference, is represented by the middle of the solid diamonds. A test of heterogeneity between the trials within a subgroup is given below the summary statistics.

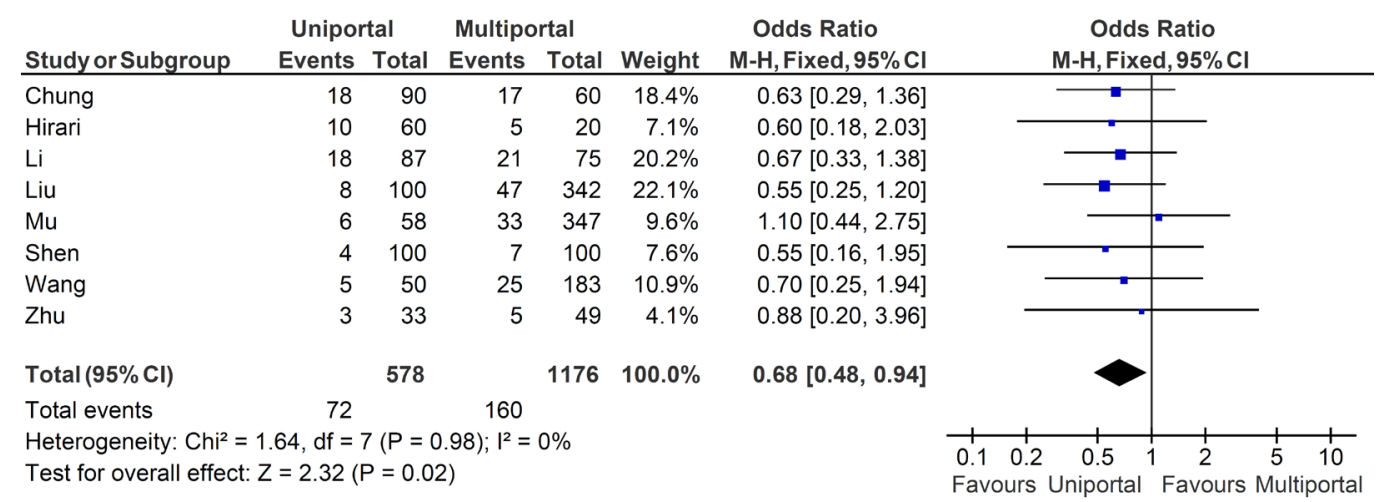

Figure 4 Forest plot of overall morbidity for uniportal and multiportal groups. The estimate of the mean difference of each study corresponds to the middle of the squares and the horizontal line shows the $95 \%$ confidence interval (CI). On each line, the mean and standard deviations are shown for both treatment groups. The sum of the statistics, along with the summary standardized mean difference, is represented by the middle of the solid diamonds. A test of heterogeneity between the trials within a subgroup is given below the summary statistics. 


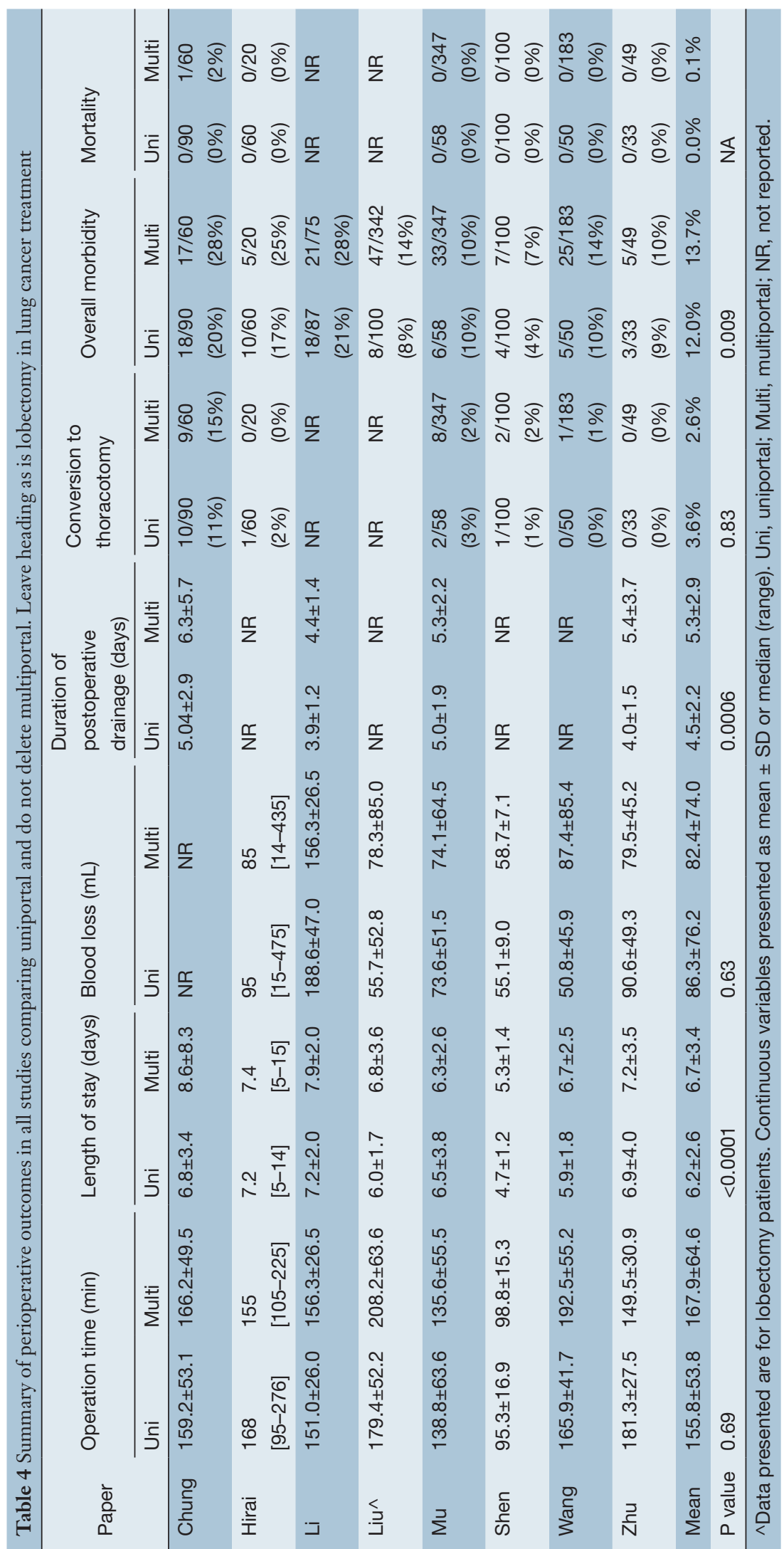

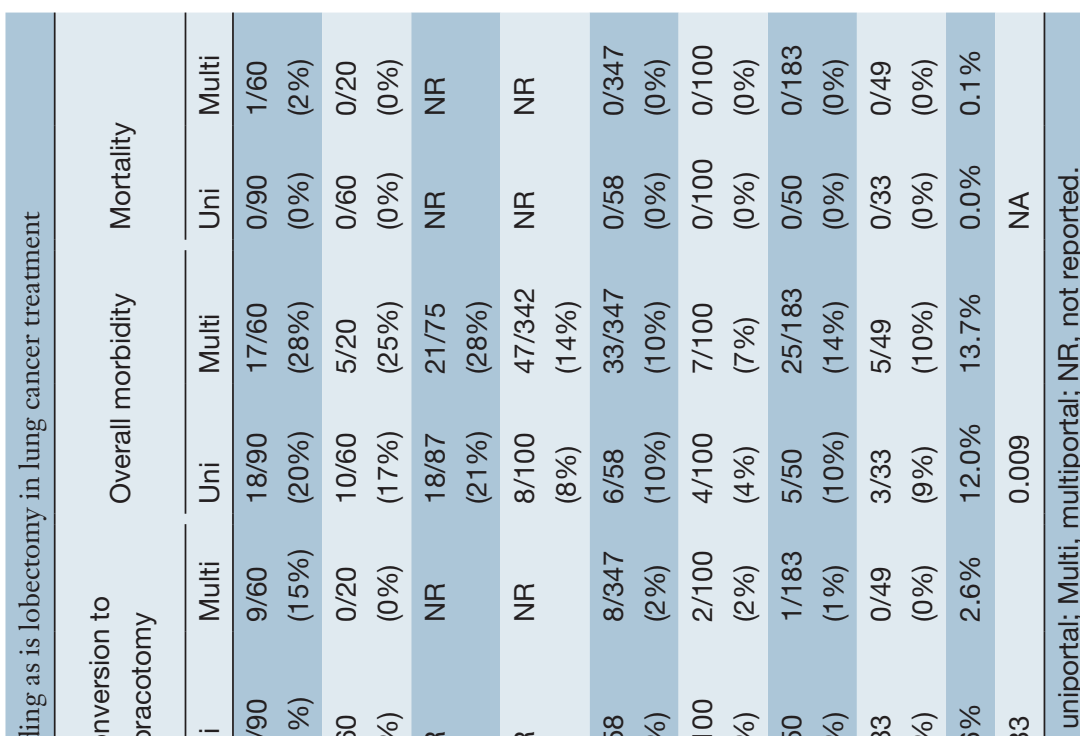


overall morbidity ( $12.0 \%$ vs. $13.7 \%)$ compared to multiportal VATS. However, these findings must be considered in the context of their clinical relevance, as the improvements were only minor. Furthermore, the benefits of uniportal VATS were even less significant when propensity-matched data were meta-analyzed. Results also demonstrated that there were no significant differences between the two treatment modalities in regard to the number of lymph nodes dissected, operative time or rates of conversion to open thoracotomy. Overall, these findings suggested that uniportal VATS can be performed with relatively similar or improved perioperative outcomes without compromising safety or oncologic principles. However, there was a paucity of long-term clinical data and equivalent oncologic efficacy cannot be ascertained based on the existing literature.

Improved pain management has been a frequently claimed benefit to the uniportal VATS approach. However, the evidence for this was limited, with only one study in the present review reporting patient pain outcomes (23). Although this study demonstrated a reduction in pain scores, as measured by Visual Analogue Score (VAS) of 1-10 on day 1 postoperatively, a recent Best Evidence Topic review of uniportal and multiport VATS surgery concluded that the single port approach made little difference in pain outcomes for patients undergoing minor thoracic surgeries (24). Based on these findings, it is clear that further studies with standardized pain management protocols are required to determine benefits of the uniportal approach for pain management. Opponents of uniportal VATS have voiced concerns that this approach may be associated with longer operative duration, worse safety outcomes and higher likelihood of conversion to open thoracotomy. However, results of the present metaanalysis have refuted these concerns, at least in the context of selected patients treated in specialized centers.

It is important to acknowledge a number of limitations when considering the results of the present systematic review. Of the eight observational studies included for meta-analysis, one was prospective (20) and all others were retrospective in design. Wang and colleagues noted that a randomized control trial could not be easily performed in this setting, but suggested further prospective studies in the future (22). In order to minimize patient selection bias due to the non-random allocation of treatment, three studies performed propensity-matched analyses to improve the matching of patients according to relevant prognostic factors (20-22). Other limitations of the systematic review included the variable reporting of conversions in different studies and variable grading of postoperative morbidities. Whilst most studies included conversion rates (from single port to multiportal VATS or from VATS to open thoracotomy) in their analysis of surgical outcomes, Wang and colleagues used conversion as one of their exclusion criteria for statistical analysis (22). Furthermore, some studies accounted for a learning curve period by excluding the initial patients who underwent uniportal VATS from analysis in order to exclude the learning curve (21). These arbitrary exclusions may have had an impact on the surgical outcomes of the uniportal VATS treatment arm.

In conclusion, the present study was the first to metaanalyze clinical outcomes of uniportal VATS versus multiportal VATS in the treatment of lung cancer. Results suggested that uniportal VATS was associated with a statistically significant reduction in the duration of chest tube drainage, in-hospital stay and overall morbidity, but these improvements may only be minor in the clinical setting. Future studies should aim to standardize clinical outcomes with longer follow-up to assess the oncologic efficacy of the uniportal approach.

\section{Acknowledgements}

None.

\section{Footnote}

Conflicts of Interest: The authors have no conflicts of interest to declare.

\section{References}

1. Cao C, Manganas C, Ang SC, et al. Video-assisted thoracic surgery versus open thoracotomy for non-small cell lung cancer: a meta-analysis of propensity score-matched patients. Interact Cardiovasc Thorac Surg 2013;16:244-9.

2. Yan TD, Black D, Bannon PG, et al. Systematic review and meta-analysis of randomized and nonrandomized trials on safety and efficacy of video-assisted thoracic surgery lobectomy for early-stage non-small-cell lung cancer. J Clin Oncol 2009;27:2553-62.

3. Cao C, Gupta S, Chandrakumar D, et al. Meta-analysis of intentional sublobar resections versus lobectomy for early stage non-small cell lung cancer. Ann Cardiothorac Surg 2014;3:134-41.

4. Gonzalez-Rivas D, Paradela M, Fieira E, et al. Single- 
incision video-assisted thoracoscopic lobectomy: initial results. J Thorac Cardiovasc Surg 2012;143:745-7.

5. Gonzalez-Rivas D, Paradela M, Fernandez R, et al. Uniportal video-assisted thoracoscopic lobectomy: two years of experience. Ann Thorac Surg 2013;95:426-32.

6. Gonzalez-Rivas D, Fieira E, Delgado M, et al. Is uniportal thoracoscopic surgery a feasible approach for advanced stages of non-small cell lung cancer? J Thorac Dis 2014;6:641-8.

7. Lin Z, Xi J, Xu S, et al. Uniportal video-assisted thoracic surgery lobectomy in semiprone position: primary experience of 105 cases. J Thorac Dis 2015;7:2389-95.

8. Tamura M, Shimizu Y, Hashizume Y. Pain following thoracoscopic surgery: retrospective analysis between single-incision and three-port video-assisted thoracoscopic surgery. J Cardiothorac Surg 2013;8:153.

9. Mier JM, Chavarin A, Izquierdo-Vidal C, et al. A prospective study comparing three-port video-assisted thoracoscopy with the single-incision laparoscopic surgery (SILS) port and instruments for the video thoracoscopic approach: a pilot study. Surg Endosc 2013;27:2557-60.

10. Kang do K, Min HK, Jun HJ, et al. Early outcomes of single-port video-assisted thoracic surgery for primary spontaneous pneumothorax. Korean J Thorac Cardiovasc Surg 2014;47:384-8.

11. Yang HC, Cho S, Jheon S. Single-incision thoracoscopic surgery for primary spontaneous pneumothorax using the SILS port compared with conventional three-port surgery. Surg Endosc 2013;27:139-45.

12. Salati M, Brunelli A, Xiumè F, et al. Uniportal videoassisted thoracic surgery for primary spontaneous pneumothorax: clinical and economic analysis in comparison to the traditional approach. Interact Cardiovasc Thorac Surg 2008;7:63-6.

13. Ocakcioglu I, Alpay L, Demir M, et al. Is single port enough in minimally surgery for pneumothorax? Surg Endosc 2016;30:59-64.

14. Chen PR, Chen CK, Lin YS, et al. Single-incision thoracoscopic surgery for primary spontaneous pneumothorax. J Cardiothorac Surg 2011;6:58.

Cite this article as: Harris CG, James RS, Tian DH, Yan TD, Doyle MP, Gonzalez-Rivas D, Cao C. Systematic review and meta-analysis of uniportal versus multiportal video-assisted thoracoscopic lobectomy for lung cancer. Ann Cardiothorac Surg 2016;5(2):76-84. doi: 10.21037/acs.2016.03.17
15. Moher D, Liberati A, Tetzlaff J, et al. Preferred reporting items for systematic reviews and meta-analyses: the PRISMA statement. BMJ 2009;339:b2535.

16. Chung JH, Choi YS, Cho JH, et al. Uniportal videoassisted thoracoscopic lobectomy: an alternative to conventional thoracoscopic lobectomy in lung cancer surgery? Interact Cardiovasc Thorac Surg 2015;20:813-9.

17. Hirai K, Takeuchi S, Usuda J. Single-incision thoracoscopic surgery and conventional video-assisted thoracoscopic surgery: a retrospective comparative study of perioperative clinical outcomes $†$. Eur J Cardiothorac Surg 2016;49 Suppl 1:i37-i41.

18. Li C, Ma H, He J, et al. Clinical analysis of thoracoscopic lobectomy in the treatment of peripheral lung cancer with single utility port. Zhongguo Fei Ai Za Zhi 2013;16:487-91.

19. Liu CC, Shih CS, Pennarun N, et al. Transition from a multiport technique to a single-port technique for lung cancer surgery: is lymph node dissection inferior using the single-port technique?†. Eur J Cardiothorac Surg 2016;49 Suppl 1:i64-i72.

20. Mu JW, Gao SG, Xue Q, et al. A Matched Comparison Study of Uniportal Versus Triportal Thoracoscopic Lobectomy and Sublobectomy for Early-stage Nonsmall Cell Lung Cancer. Chin Med J (Engl) 2015;128:2731-5.

21. Shen Y, Wang H, Feng M, et al. Single- versus multipleport thoracoscopic lobectomy for lung cancer: a propensity-matched study†. Eur J Cardiothorac Surg 2016;49 Suppl 1:i48-i53.

22. Wang BY, Liu CY, Hsu PK, et al. Single-incision versus multiple-incision thoracoscopic lobectomy and segmentectomy: a propensity-matched analysis. Ann Surg 2015;261:793-9.

23. Zhu Y, Liang $M, W u ~ W$, et al. Preliminary results of single-port versus triple-port complete thoracoscopic lobectomy for non-small cell lung cancer. Annals of translational medicine 2015;3:92.

24. Akter F, Routledge T, Toufektzian L, et al. In minor and major thoracic procedures is uniport superior to multiport video-assisted thoracoscopic surgery? Interactive CardioVascular and Thoracic Surgery 2015;20:550-5 
Appendix

\begin{tabular}{|c|c|c|c|c|c|c|c|c|c|c|}
\hline Paper & \multicolumn{2}{|c|}{ COPD } & \multicolumn{2}{|c|}{ Diabetes } & \multicolumn{2}{|c|}{ Tuberculosis } & \multicolumn{2}{|c|}{ Hypertension } & \multicolumn{2}{|c|}{ CAD } \\
\hline Chung & 5 (6\%)\# & 4 (7\%)\# & $\begin{array}{l}11 \\
(12 \%)\end{array}$ & $\begin{array}{l}11 \\
(18 \%)\end{array}$ & $\begin{array}{l}6 \\
(7 \%)\end{array}$ & $\begin{array}{l}6 \\
(10 \%)\end{array}$ & $\begin{array}{l}24 \\
(27 \%)\end{array}$ & $\begin{array}{l}24 \\
(40 \%)\end{array}$ & $\begin{array}{l}4 \\
(4 \%)\end{array}$ & $\begin{array}{l}3 \\
(5 \%)\end{array}$ \\
\hline Liu^ & $5(5 \%)$ & 27 (78\%) & $11(11 \%)$ & 40 (11.7\%) & $6(6 \%)$ & $5(2 \%)$ & NR & NR & NR & NR \\
\hline $\mathrm{Mu}$ & NR & NR & $6(10 \%)$ & 35 (10\%) & NR & NR & $14(24 \%)$ & $117(34 \%)$ & $9(16 \%)$ & $34(10 \%)$ \\
\hline Shen & NR & NR & NR & NR & NR & NR & NR & NR & NR & NR \\
\hline Wang & NR & NR & NR & NR & NR & NR & NR & NR & NR & NR \\
\hline
\end{tabular}

